\title{
Index locorum
}

\section{Accio}

Acc. trag. 223-225 R3: 209

\section{Aezio (ed. Mansfeld-Runia)}

Aët. 1.7.4-10: 141

Aët. 2.3: 130, 196

Aët. 2.4: 196

Aët. 2.4.10: 16

Aët. 2.4.11: 6

\section{Agostino}

Aug. c. Iul. 4.12 .60 t. 10 p. 612 Ben.: 160 Aug. civ. 7.8: 186

Aug. civ. 7.13: 19, 130

Aug. civ. 7.23 (= Varro, antiquitates rerum divinarum fr. 227 Cardauns): 19, 130

Aug. civ. 22.28: 19

\section{Anassimandro \\ D.-K. 12 A11: VII \\ D.-K. 12 A14: VII \\ D.-K. 12 A17: 2 \\ D.-K. 12 A27: 2 \\ D.-K. 12 A28: 2 \\ D.-K. 12B2: VII \\ D.-K. 12B3: VII}

Anonimo Sul sublime

subl. 7.1: 256

subl. 9.6: 69, 100, 261

subl. 9.8: 261

subl. 15.4: 190

subl. 35.3-5: 231

\section{Apollonio Rodio}

Ap. Rhod. Argon. 4.597: 189

\section{Apuleio}

Apul. mund. 27: 114

\section{Arato}

Aratus phaen. 2-4: 97
Aratus phaen. 10-14: 97

Aratus phaen. 20-23: 9, 114

\section{Aristofane}

Ar. Lys. 125-127: 274

\section{Aristotele}

Arist. de philosophia fr. 13c Ross: 313, 334

Arist. de philosophia fr. 18 Ross: 9, 92, 120, 123, 228, 230, 247

Arist. part. an. 686a 28-29: 161

Arist. part. an. 687a 23: 161

Arist. cael. 1(A)10.279b5-30: 3

Arist. cael. 1(A)10.279b5-284b4: 10

Arist. cael. 1(A)10.279b30-280a10: 9

Arist. cael. 1(A)12.281b20-282a4: 10

Arist. cael. 1(A)12.282a25-283a3: 10

Arist. cael. 2(B)1.283b25-284a5: 11

Arist. cael. 2(B)1.284a20: 316

Arist. cael. 2(B)1.284a20-25: 10

Arist. phys. 2.6.198a5-13: 117

Arist. phys. 8.250b11-253a2: 10

Arist. mete. 353a15: 9

Arist. mete. 355a1: 44

Arist. mete. 356b13-15: 292

Arist. gen. corr. B6.333a 35: 296

Arist. sens. 2.437b23: 295

\section{pseudo-Aristotele}

ps.-Arist. mund. 391a1-b8: 310

ps.-Arist. mund. 391b5-8: 314

ps.-Arist. mund. 391b17: 114-115

ps.-Arist. mund. 391b19-392a5: 114

ps.-Arist. mund. 396b20: 115

ps.-Arist. mund. 396b32: 219, 316

ps.-Arist. mund. 396b20-397a1: 228

ps.-Arist. mund. 397a27-b1: 219, 316

ps.-Arist. mund. 397b1-8: 316

ps.-Arist. mund. 397b5-7: 316

ps.-Arist. mund. 398b10-15: 114

ps.-Arist. mund. 398b 12-16: 318

ps.-Arist. mund. 399b10-15: 128

ps.-Arist. mund. 400a3-7: 132 
ps.-Arist. mund. 400a3-14: 315

ps.-Arist. mund. 400a25-400b6: 231, 320

ps.-Arist. mund. 400b1-6: 242, 320

ps.-Arist. mund. 400b6-13: 319

ps.-Arist. mund. 400b10-30: 242

ps.-Arist. mund. 400b13-27: 318

ps.-Arist. mund. 400b27-28: 132

ps.-Arist. mir. ausc. 38: 231

ps.-Arist. mir. ausc. 40: 231

\section{Arriano}

Arr. Epict. diss. 1.16.21: 141

\section{Bacchilide}

Bacchyl. 5.160-162: 161

\section{Catone}

Cato agr. praef. 2: 277

\section{Catullo}

Catull. 5.2: 279

Catull. 64.290-291: 189

\section{Censorino}

Cens. 9.1-3: 19

Cens. 17.5: 22

Cens. 21.1-5: 19

\section{Cicerone}

Cic. acad. 1.24-29: 21

Cic. acad. 1.27-28: 28-29, 130

Cic. acad. 1.29: 141

Cic. Arat. phaen. fr. 2 Buescu: 20, 123-125, 139, 164, 235, 250, 290, 337

Cic. Arat. phaen. fragm. max. 296: 114

Cic. carm. frg. 6 (= fr. 11 Traglia; 10 C.; 2 S.) 1-3: 171

Cic. carm. frg. 6 (= fr. 11 Traglia; 10 C.; 2 S.) 1-10: 229

Cic. carm. frg. 6 (= fr. 11 Traglia; 10 C.; 2 S.) 23-53: 24

Cic. carm. frg. 6 (= fr. 11 Traglia; 10 C.; 2 S.) 34: 229

Cic. carm. frg. 6 (= fr. 11 Traglia; 10 C.; 2 S.) 36-37: 289

Cic. carm. frg. 6 (= fr. 11 Traglia; 10 C.; 2 S.) 47-48: 229
Cic. Att. 13.33: 281

Cic. Att. 13.39.2: 137

Cic. div. 2.40: 133, 336

Cic. div. 2.98: 22

Cic. Luc. 55: 5

Cic. Luc. 116: 53

Cic. Luc. 116-124: 53, 128

Cic. Luc. 118-120: 20, 122-124

Cic. Luc. 120: 159

Cic. nat. deor. 1.18-24: 162

Cic. nat. deor. 1.19: 115

Cic. nat. deor. 1.20: 137, 139, 182

Cic. nat. deor. 1.22: 144, 146

Cic. nat. deor. 1.23: 144, 146

Cic. nat. deor. 1.25-41: 137

Cic. nat. deor. 1.26: 44

Cic. nat. deor. 1.33: 128

Cic. nat. deor. 1.43: 138

Cic. nat. deor. 1.59: 137

Cic. nat. deor. 1.66: 117

Cic. nat. deor. 1.72: 13

Cic. nat. deor. 1.90-91: 117

Cic. nat. deor. 2.23-28: 56

Cic. nat. deor. 2.30-31: 181

Cic. nat. deor. 2.37: 269

Cic. nat. deor. 2.40: 46, 55

Cic. nat. deor. 2.42: 44

Cic. nat. deor. 2.49: 186

Cic. nat. deor. 2.56: 117

Cic. nat. deor. 2.73-75: 242

Cic. nat. deor. 2.88: 115

Cic. nat. deor. 2.93-94: 117

Cic. nat. deor. 2.98-104: 155

Cic. nat. deor. 2.115-118: 52, 55

Cic. nat. deor. 2.118: 46

Cic. nat. deor. 2.153: 160

Cic. nat. deor. 2.154: 132, 154-155

Cic. nat. deor. 2.154-55: 132, 146

Cic. nat. deor. 2.161: 159

Cic. nat. deor. 2.167: 146, 151, 159

Cic. nat. deor. 3.23: 21, 129

Cic. nat. deor. 3.28-34: 54

Cic. nat. deor. 3.29-31: 181

Cic. nat. deor. 3.37: 57

Cic. nat. deor. 3.76: 189

Cic. nat. deor. 3.86: 159, 273

Cic. off. 2.16: 18 
Cic. off. 3.94: 189, 191

Cic. de orat. 2.155: 86

Cic. rep. 2.26: 282, 306

Cic. rep. 6.23: 20, 189

Cic. Tim. 9: 115

Cic. Tim. 41: 161

Cic. Tusc. 3.32: 307

Cic. Tusc. 4.6: 249

Cic. Tusc. 5.117-118: 147

\section{Clemente Alessandrino}

Clem. Al. strom. 5.81: 111, 296

Clem. Al. strom. 6.5.1: 22

\section{Cleomede}

Cleomedes 1.1.81-100: 60

Cleomedes 1.1.258: 49

Cleomedes 2.1.426-451: 121

\section{Columella}

Colum. 2.1: 287-288

Colum. 3.15: 277

Colum. 10.215-216: 264

\section{Cornuto}

Cornutus theol. Graec. 6 (S.6, 3-6 Lang): 96

\section{Demetrio Falereo}

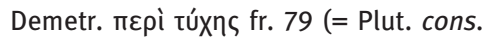
Apoll. 104a11): 113

\section{Demetrio Lacone}

Demetrius Laco P.Herc 831 col. 8.7-13 N 75 Körte (1890) 583: 303, 312

\section{Democrito}

D.-K. 68 A37 (= 293 Luria): 4, 81

D.-K. 68 A40: VII, 5, 75, 81

D.-K. 68 A84 (= 353 Luria): 5

D.-K. 68B5: 82

D.-K. 68B5.2: 82

Fr. 351 Luria (= Philo aet. 3): 5

Fr. 354 Luria (= Plut. quaest. conv. 8.9.3

p. 733c): 5

\section{Dicearco di Messina}

Fr. 47 Wehrli: 18

Fr. 48 Wehrli: 18

\section{Diogene di Enoanda}

NF 39(= fr. 20 Smith): 145, 152, 163, 248

NF 40 (= fr. 21 Smith): 152, 163

NF 54 (= fr. 22 Smith): 152-153

NF 126: 144

NF 127: 144

NF 155: $137-138,144$

NF 182: 206

\section{Diogene Laerzio}

Diog. Laert. 7.151-154: 207

\section{Diodoro Siculo}

Diod. Sic. 5.23.2-3: 191-195

\section{Empedocle}

D.-K. 31 A30: 3

D. - K. 31 A52: VII

D.-K. 31 A70: 80

D.-K. 31 A72C: 289

D.-K. 31B17: 3

D.-K. 31B18: 3

D. - K. 31B20: 300

D.-K. 31B21: 3

D.-K. 31B22: 3

D.-K. 31B26: 300

D.-K. 31B27: 111, 207, 293

D.-K. 31B37: 296

D.-K. 31B38: 201

D.-K. 31B51: 294

D.-K. 31B62: 294

D.-K. 31B84: 295

D.-K. 31B110: 295

D.-K. 31B112: 292

D.-K. 31B115: 300

D.-K. 31B129: 313

D.-K. 31B133: 111, 296

Papiro di Strasburgo

fr. a(ii) vv. 296-297: 296-297

\section{Ennio}

Enn. fr. inc. 16 V.: 186

Enn. ann. fr. 6 Sk.: 289 
Enn. ann. fr. 7 Sk.: 289

Enn. ann. fr. 225 Sk.: 291

Enn. ann. fr. 226 Sk.: 291

Enn. ann. fr. 258 Sk.: 112

Enn. ann. fr. 481 Sk.: 276

Enn. ann. fr. 577 Sk.: 306

\section{Epicuro}

Epic. ep. Hdt. 39: 257

Epic. ep. Hdt. 45: 27

Epic. ep. Hdt. 60: 100

Epic. ep. Hdt. 73-74: 14, 16, 27, 84, 180

Epic. ep. Hdt. 78: 70, 202

Epic. ep. Hdt. 80: 232

Epic. ep. Moen. 124: 256, 308

Epic. ep. Moen. 133: 155

Epic. ep. Moen. 135: 256, 308

Epic. ep. Pyth. 85: 267

Epic. ep. Pyth. 87: 202

Epic. ep. Pyth. 88-90: 15, 27, 80, 83, 133, 219, 331, 335

Epic. ep. Pyth. 90: 116

Epic. ep. Pyth. 93: 46

Epic. fr. 221 Usener: 308

Epic. fr. 299 Usener: 100

Epic. fr. 305 Usener: 16, 76, 84-85, 236

Epic. fr. 361 Usener: 141, 162-163

Epic. fr. 499 Usener: 147

Epic. RS 11: 267

Epic. RS 13: 32

Epic. RS 16: 116

Epic. RS 31: 324

Epic. Sent. Vat. 10: 268, 312

Epic. Sent. Vat. 27: 266

Epic. Sent. Vat. 31: 17

Epic. Sent. Vat. 32: 268

Epic. Sent. Vat. 33: 276-277

\section{Eraclito}

D.-K. 22 A5: VIII, 2

D.-K. 22 A10: VIII, 2

D.-K. 22 A13: 2

D.-K. 22 A65: 2

D.-K. 22B30: VIII

\section{Esiodo}

Hes. theog. 700-705: 68
Hes. theog. 842: 69

Hes. theog. 855: 294

Hes. op. 143-201: 279

Esopo

Aes. 8: 292

\section{Euripide}

Eur. fr. $420 \mathrm{~N}$ (Ino): 112-113

Eur. fr. 779 N (Phaethon): 190

Eur. fr. 839 N (Chrysippus): 175, 316

Eur. Hec. 284-285: 113

Eur. HF 508-510: 112

\section{Eusebio}

Euseb. praep. evang. 14.27.9: 312

\section{Filodemo}

Phld. de dis III coll. IX 36 - X 6: 132

Phld. de morte IV col. XXXVI 17-26: 26

Phld. de morte IV col. XXXVIII 17-18: 256, 284

Phld. de pietate II Obbink 126-7 (P.Herc. 1428 coll. IV-V): 129,137

Phld. rhet. P.Herc. 1015/832 col. LVI 15-20: 321

Phld. epigr. 29 Sider (= anth. Gr. 9.412.78): 284

\section{Filolao}

D.-K.44 A18: 7

\section{pseudo-Filolao}

ps. Philolaus de anima 150.12-20: 6

\section{Filone Alessandrino}

Philo aet. 8-9: 12, 250

Philo aet. 10 (= Arist. de philosophia fr. 18 Ross): 9

Philo aet. 12: 6

Philo aet. 14-15: 9

Philo aet. 21-27: 227

Philo aet. 28-34: 54, 67, 247

Philo aet. 45-47: 121, 123

Philo aet. 47: IX, 121

Philo aet. 55-75: 84-94, 246, 325

Philo aet. 72-73: 90, 121-122, 324, 328 
Philo aet. 76-77: 19

Philo aet. 78: 138

Philo aet. 110-112: 121

Philo aet. 114-116: 40, 60

Philo aet. 117-149 (= Theophr. fr. 184

FHS\&G): XV, 16-17, 164-182, 189, 245246

Philo ebr. 199: 113, 117

Philo prov. 1.13-20: 182

Philo prov. 1.17-19: 249

Philo prov. 1.37: 159

Philo prov. 2.56-65: 239

Philo prov. 2.84: 151

Philo prov. 2.87: 159

Philo prov. 2.90: 113, 160, 231, 239, 249, 339

Philo prov. 2.91: 159

Philo prov. 2.92-94: 159

\section{Filopono}

Johannes Philoponus aet. mund. 145.13147.257: 9

\section{Frontone}

Fronto de feriis Alsiensibus 3.1 p. 227.8: 255

\section{Giustino martire}

Just. apol. 1.20.1: 22

\section{Gromatici veteres}

Grom. 350.17-351.11 Lachmann (= Profezia di Vegoia): 22, 253, 288

\section{Lattanzio}

Lact. inst. 7.1.10: 179

Lact. inst. 7.3.13: 138

Lact. inst. 7.15.19: 22

Lact. inst. 7.18.2: 22

\section{Leucippo}

D.-K. 67 A1 (= 289 Luria): VII, 4, 81

D.-K. 67 A10 (= 281 Luria): 4

\section{Livio Andronico}

Liv. Andr. carm. frg. 23 (= 11 Morel): 112
Lucano

Lucan. 1.639-672: 24

Luciano

Lucian. Herm. 78: 284

Lucilio

Lucil. fr. 1 Marx: XII

Lucil. fr. 53 Marx: 284

Lucrezio

\section{Libro I}

Lucr. 1.1-43: 258, 291, 298-299

Lucr. 1.1: 306

Lucr. 1.4-5: 337

Lucr. 1.6: 339

Lucr. 1.21-24: 258

Lucr. 1.25-27: 314

Lucr. 1.42-43: 314

Lucr. 1.55-57: 38,333

Lucr. 1.58: 333

Lucr. 1.61: 332

Lucr. 1.62-79: XIII, 29-33, 74, 100, 122, $262-263,286,293,301,310,313-314$

Lucr. 1.68-69: 30, 69, 209, 327, 334

Lucr. 1.70-71: 328

Lucr. 1.72-74: 331

Lucr. 1.73: 331

Lucr. 1.75-77: 31, 88, 264, 312, 338

Lucr. 1.79: $32,269,328$

Lucr. 1.102-103: 27, 133

Lucr. 1.102-109: 367

Lucr. 1.107-109: 37

Lucr. 1.121: 332

Lucr. 1.231: 46,58

Lucr. 1.249: 333

Lucr. 1.250-264: 80

Lucr. 1.136-137: 254, 302

Lucr. 1.594-596: 338

Lucr. 1.635-704: 328

Lucr. 1.638-644: 91, 93, 96

Lucr. 1.676: 332

Lucr. 1.716-741: 291

Lucr. 1.722-725: 32, 226, 292

Lucr. 1.726-733: 291, 298

Lucr. 1.734-741: 292 
Lucr. 1.788: 332

Lucr. 1.827: 333

Lucr. 1.921-950: 33, 181, 302

Lucr. 1.951-1051: 38

Lucr. 1.1014-1022: 65

Lucr. 1.1018: 229

Lucr. 1.1021-1051: 38, 64, 66, 332

Lucr. 1.1038-1041: 75

Lucr. 1.1052-1117: XIII, XXIII, 26, 35-69, 244-245, 332

Lucr. 1.1052-1082: 38

Lucr. 1.1053: 39, 41, 48

Lucr. 1.1055: 48

Lucr. 1.1068-1075: 38

Lucr. 1.1083-1086: 38-39, 45-46, 50, 295

Lucr. 1.1102-1113: 58-65, 294

Lucr. 1.1105-1107: 39, 62, 81, 214

Lucr. 1.1114-1117: 70, 224, 257, 260, 262, 265, 296, 311, 325, 329, 334

\section{Libro II}

Lucr. 2.1-13: 326, 341

Lucr. 2.3-4: 304, 341

Lucr. 2.9-10: 326

Lucr. 2.16-17: 276

Lucr. 2.55-59: 329

Lucr. 2.61: 333

Lucr. 2.62-79: 286

Lucr. 2.65: 92, 317

Lucr. 2.71-76: 323

Lucr. 2.78.79: 90

Lucr. 2.167-182: 82, 96, 146, 151, 325

Lucr. 2.174: 325

Lucr. 2.181: 332, 361

Lucr. 2.220: 278

Lucr. 2.303-307: 257

Lucr. 2.328: 332

Lucr. 2.569-580: 106, 242, 271, 286, 323, 340

Lucr. 2.575-580: 318

Lucr. 2.589-660: 90, 93, 95, 288, 337

Lucr. 2.601-604: 96

Lucr. 2.606-607: 96

Lucr. 2.625: 336

Lucr. 2.644-645: 96

Lucr. 2.863: 336

Lucr. 2.991-1003: 175, 316, 337
Lucr. 2.1023-1047: 33, 74, 81, 109, 268, 325,333

Lucr. 2.1026-1039: 333

Lucr. 2.1038: 269, 325

Lucr. 2.1040-1042: 44, 85, 110

Lucr. 2.1048-1089: 74, 81, 97, 269

Lucr. 2.1067-1076: 29, 39, 332

Lucr. 2.1077-1089: 74

Lucr. 2.1087-1089: 31, 88, 333

Lucr. 2.1090-1104: 75, 97

Lucr. 2.1095-1096: 319

Lucr. 2.1105-1174: 71-98, 214, 338

Lucr. 2.1105-1149: 234, 282

Lucr. 2.1115: 81, 296, 324

Lucr. 2.1116-1117: 89, 333

Lucr. 2.1122-30: 76-79

Lucr. 2.1123: 88-89, 283, 324

Lucr. 2.1124: 89

Lucr. 2.1130: 88, 324

Lucr. 2.1131-1132: 89, 283, 325

Lucr. 2.1139-1140: 324

Lucr. 2.1144-1145: 75, 81,90,123, 175, 201, 212, 235, 241, 283, 324-325, 331, 333

Lucr. 2.1150: 28, 90

Lucr. 2.1150-1174: 75, 105, 179, 218, 240

Lucr. 2.1164-1174：271-289

\section{Libro III}

Lucr. 3.1-13: 181

Lucr. 3.14-30: 262-263, 336

Lucr. 3.15: 297, 333

Lucr. 3.16-17: 328, 331

Lucr. 3.18-24: 336,339

Lucr. 3.25-27: 100, 314, 328, 339

Lucr. 3.28-29: 30, 68, 130, 267, 329

Lucr. 3.29-30: 31

Lucr. 3.31: 332

Lucr. 3.39: 330

Lucr. 3.55-58: 304

Lucr. 3.118-120: 278

Lucr. 3.125: 336

Lucr. 3.144: 278

Lucr. 3.324: 336

Lucr. 3.348: 336

Lucr. 3.491: 278

Lucr. 3.584: 335

Lucr. 3.647-651: 282 
Lucr. 3.784-797: 128, 185, 197 Lucr. 3.806-818: XV, 29, 185, 197 Lucr. 3.830-842: XIV, 101-103, 128, 341 Lucr. 3.834-835: 102, 323 Lucr. 3.898-899: 198, 339 Lucr. 3.945: 243, 263 Lucr. 3.952-967: 272 Lucr. 3.964: 333-340 Lucr. 3.1020-1022: 338 Lucr. 3.1042-1045: 330 Lucr. 3.1043-1044: 244 Lucr. 3.1077: 198, 327 Lucr. 3.1081: 327 Lucr. 3.1085-1086: 117, 198, 327 Lucr. 3.1085-1094: 104-105 Lucr. 3.1090: 105, 336 Lucr. 3.1091: XIV, 105, 198

\section{Libro IV}

Lucr. 4.135: 332

Lucr. 4.153: 306 Lucr. 4.506: 336 Lucr. 4.903-906: 318 Lucr. 4.942: 335 Lucr. 4.1133: 147 Lucr. 4.1239: 278 Lucr. 4.1279: 325 Lucr. 4.1283-1287: 105-106

\section{Libro V}

Lucr. 5.2-7: 267

Lucr. 5.54: 333

Lucr. 5.65-66: 340

Lucr. 5.77: 116, 333

Lucr. 5.83-87: 330

Lucr. 5.86-88: 334

Lucr. 5.88-90: 338

Lucr. 5.91-109: XIV, 108-117, 335

Lucr. 5.91-96: 110, 201, 296, 324, 331

Lucr. 5.92-94: 109

Lucr. 5.100: 333

Lucr. 5.101-103: 111, 201, 296

Lucr. 5.110-113: 112

Lucr. 5.113: 32, 297

Lucr. 5.114-116: 31, 119, 128

Lucr. 5.119-120: 136, 164, 290, 328, 332, 334
Lucr. 5.122-145: XIV, 126-130, 248

Lucr. 5.128-141: 128,185, 197

Lucr. 5.146-155: 14, 131-133, 146, 154, 162, 315,336

Lucr. 5.156-194: 125, 133-147

Lucr. 5.157-159: 323

Lucr. 5.174-180: XV, 146-147

Lucr. 5.195-234: XV, 125, 148-164

Lucr. 5.207: 340

Lucr. 5.222-234: 150-151, 161, 326-327

Lucr. 5.232-234: 93

Lucr. 5.235-305: 166-175, 247

Lucr. 5.273-280: 170-171, 249

Lucr. 5.281-305: 170, 328

Lucr. 5.295: 328

Lucr. 5.306-323: 172-175, 340

Lucr. 5.311: 331

Lucr. 5.315-317: 173, 325, 335

Lucr. 5.318-321: 109, 173-174, 182, 324, 337

Lucr. 5.330-331: 177, 332-333, 337

Lucr. 5.335-336: XV, 180-181, 234, 244

Lucr. 5.337: 177, 189, 217, 234

Lucr. 5.338-350: 216

Lucr. 5.345-350: 182, 312, 326

Lucr. 5.346-347: 203, 212, 326

Lucr. 5.347-349: 16

Lucr. 5.351-379: 29, 164, 183-186, 197, 336-337

Lucr. 5.351-363: XV, 29, 185

Lucr. 5.351: 323

Lucr. 5.352-355: 333

Lucr. 5.356-358: 329

Lucr. 5.366-368: 337

Lucr. 5.373-375: 29, 65, 186, 199, 328. 337

Lucr. 5.380-415: 164, 183, 186, 195, 211 , 225, 235-236, 326, 329

Lucr. 5.380-383: 183, 323

Lucr. 5.392-395: 190, 323

Lucr. 5.397-398: 189, 192, 264, 340

Lucr. 5.416-508: 199-201, 332-333

Lucr. 5.419-420: 220, 334

Lucr. 5.436-437: 331

Lucr. 5.523-525: 46

Lucr. 5.526-533: 236, 348

Lucr. 5.534-565: XXIII, 40

Lucr. 5.550: 335 
Lucr. 5.727: 22

Lucr. 5.780: 179

Lucr. 5.807-836: 77-78

Lucr. 5.818: 179, 333

Lucr. 5.826-836: 90, 179, 337

Lucr. 5.827: 90, 284

Lucr. 5.831: 333

Lucr. 5.943: 179

Lucr. 5.960: 327

Lucr. 5.1203: 255, 270, 280, 303, 330

Lucr. 5.1213-1214: 246, 280, 325

Lucr. 5.1215-1217: 18, 219, 316, 323, 335

Lucr. 5.1226-1235: 102, 156, 218, 305, 339340

Lucr. 5.1233: 156, 204, 340

Lucr. 5.1233-1235: 156, 210, 340

Lucr. 5.1236-1240: 212, 306

Lucr. 5.1238-1240: 320

Lucr. 5.1423-1424: 278

\section{Libro VI}

Lucr. 6.7: 324

Lucr. 6.43-46: 202-203

Lucr. 6.48-55: 202-204

Lucr. 6.50-67: 249

Lucr. 6.64-66: 338

Lucr. 6.96-159: 203

Lucr. 6.121-129: 209, 330

Lucr. 6.121-123: 209-210, 340

Lucr. 6.130-131: 205

Lucr. 6.151: 335

Lucr. 6.160-218: 203

Lucr. 6.219-422: 203

Lucr. 6.242: 331

Lucr. 6.249-252: 210

Lucr. 6.250-294: 204

Lucr. 6.251-252: 210

Lucr. 6.253-255: 211, 330

Lucr. 6.285-288: 210, 327, 340

Lucr. 6.289-292: 338

Lucr. 6.374-378: 211

Lucr. 6.379-422: 228

Lucr. 6.380-382: 229

Lucr. 6.474: 278

Lucr. 6.485-494: 234

Lucr. 6.489-491: 338

Lucr. 6.535-607: 203, 212-219, 337
Lucr. 6.535-542: 212-213

Lucr. 6.543-551: 212-214, 217

Lucr. 6.546: 213, 335

Lucr. 6.552-556: 213

Lucr. 6.557-576: 216, 219, 329

Lucr. 6.557-595: 214

Lucr. 6.565-566: 213, 216, 323, 338

Lucr. 6.572-573: 213

Lucr. 6.573-575: 228

Lucr. 6.577-590: 213

Lucr. 6.578: 340

Lucr. 6.589-593: 235

Lucr. 6.591-595: 213

Lucr. 6.592: 340

Lucr. 6.594-595: 205

Lucr. 6.596-607: 65, 213, 218, 303

Lucr. 6.598: 65, 335, 338

Lucr. 6.599: 329, 338

Lucr. 6.602: 18, 66, 219, 227-228, 246, 303, 316, 323, 335

Lucr. 6.606: $66,329,339$

Lucr. 6.607: 26, 65-66, 201, 219-220

Lucr. 6.639-702: 203-205, 220-226, 238, 297

Lucr. 6.639-645: 226, 302-303, 329

Lucr. 6.641-679: 183, 326, 334

Lucr. 6.642-646: 297, 338

Lucr. 6.644-646: 303

Lucr. 6.647-654: 223, 331

Lucr. 6.647-648: 205, 227, 332

Lucr. 6.649-652: 102, 326

Lucr. 6.653-654: 298, 326

Lucr. 6.655-669: 205, 326

Lucr. 6.665: 225, 334

Lucr. 6.666-667: 226

Lucr. 6.668: 224, 235

Lucr. 6.669: 224, 234

Lucr. 6.671-672: 224, 327

Lucr. 6.673-679: 224

Lucr. 6.679: 227, 329

Lucr. 6.703-711: 232

Lucr. 6.703-737: 238

Lucr. 6.738-839: 238

Lucr. 6.840-905: 238

Lucr. 6.906-1089: 238

Lucr. 6.1090-1286: 238

Lucr. 6.1090: 335 
Lucr. 6.1099: 241

Lucr. 6.1101-1103: 241

Lucr. 6.1121: 241

Lucr. 6.1140-1142: 241

Lucr. 6.1277-1286: 257, 299, 323

Lucr. 6.1282-1283: 300, 336

Lucr. 6.1285-1286: 243, 299, 300, 319

\section{Manilio}

Manil. 1.149-159: 57-58

Manil. 5.742-5: 65

\section{Marziale}

Mart. 3.82: 279

\section{Menandro}

Men. frg. 372 K.-A.: 118

\section{Metrodoro di Chio}

D.-K. 70 A6: 75, 234

\section{Omero}

Hom. Il. 5.646: 328

Hom. Il. 6.448-449: 112

Hom. Il. 13.18-19: 262

Hom. Il. 13.27-29: 262

Hom. Il. 15.623-629: 302

Hom. Il. 17.200: 274

Hom. Il. 18.483-484: 324

Hom. Il. 20.61-65: 100

Hom. Il. 20.63-65: 210, 339

Hom. Il. 21.388: 261

Hom. Od. 3.42-45: 315

Hom. Od. 20.13: 276

Hom. Od. 24.226-234: 273

\section{Oracoli Sibillini}

Oracula Sibyllina 3.341-342: 23

\section{Orazio}

Hor. ars 156-157: 272, 277

Hor. ars 169-174: 272

Hor. ars 464-466: 293

Hor. epist. 1.7.84: 279

Hor. carm. 3.30.1-5: 174

Hor. sat. 1.28: 273

\section{Ovidio}

Ov. am. 1.15.23-24: 290

Ov. met. 1.750-770: 189

Ov. met. 2.1-400: 189, 191

Ov. trist. 2.424-425: XXI

\section{Pacuvio}

Pacuv. trag. 86-92 (= 79-80 Schierl): 109 , 175

Pacuv. trag. $372-375 R^{3}$ (= 262 Schierl): 118

Pacuv. trag. 411-416 $\mathrm{R}^{3}$ (= 496 Schierl): 204

\section{Panezio}

Fr. 68 Van Straaten: XII

\section{Parmenide}

D.-K. 28 A23: VII

D.-K. 28 A25: VII

D.-K. 28 A36: VII

D.-K. 28B8: 115

D.-K. $28 B 12$ v. 3: 117

\section{Platone}

Pl. Criti. 109d: 8

Pl. Criti 111a: 8

Pl. Gorg. 507e-508a: 132

Pl. leg. 677a: 8

Pl. leg. 776b: 90

Pl. leg. $886 \mathrm{~d}-\mathrm{e}: 119,132$

Pl. leg. 889a-c: 117

Pl. leg. $890 \mathrm{~b}-\mathrm{c}: 119$

Pl. Menex. 237e: 80

Pl. plt. 269c-274e: 3

Pl. Phdr. 247c-249c: 313

Pl. Phd. 108e-109a: 45

Pl. Prt. 320c-322d: 93

Pl. resp. 7.514b-520a: 313

Pl. soph. 242d: VII

Pl. soph. 246a: 119

Pl. Ti. 21e-25d: 7

Pl. Ti. 23a: 178

Pl. Ti. 23c-25d: 8

Pl. Ti. 25 c-d: 178

Pl. Ti. 29d: 115

Pl. Ti. 30b-c: 130

Pl. Ti. 32c-33b: 226 
Pl. Ti. 32d: 115

Pl. Ti. 36d-37a: 130

Pl. Ti. 41a-41b: 7

PI. Ti. 40c-42d: 32

Pl. Ti. 62e1-63a2: 41

Pl. Ti. 78e-81d: 76

Pl. Ti. 81a4-d4: 76

\section{Plauto}

Plaut. Amph. 496: 279

Plaut. Asin. 403-406: 275

Plaut. Bacch. 303-305: 274

Plaut. Capt. 133-135: 283

Plaut. Merc. 290-291: 277

Plaut. Merc. 314: 277

Plaut. Merc. 599-600: 274

Plaut. Merc. 838-841: 276

Plaut. Mil. 627-628: 284

Plaut. Persa 266-267: 277

Plaut. Poen. 360: 276

Plaut. Rud. 523: 277

Plaut. Stich. 648: 283

Plaut. Trin. 280-300: 280

Plaut. Trin. 296: 277

Plaut. Trin. 1169: 274

Plinio il vecchio

Plin. nat. 2.10-11: 59, 62

Plin. nat. 2.89-248: 207

\section{Plutarco}

Plut. comm. not. 31, 1075a: IX

Plut. fac. 923e-927a: 43-44, 48, 52-55, 245

Plut. fac. 927 b-c: 54

Plut. Stoic. rep. 1054b-d: 13, 52

Plut. Stoic. rep. 1051d-e: 157

Plut. vit. Rom. 12.4-6: 22

pseudo-Plutarco

ps.-Plut. placit. 879a10-b6: 151, 163

ps.-Plut. placit. $886 \mathrm{e} 1-5: 56$

\section{Polistrato}

Polystratus, de contemptu coll.12.21-13.24: $155,157,163$

\section{Properzio}

Prop. 3.5.31: 290

Quintiliano

Quint. inst. 3.5.5.12: 23

Quint. inst. 7.2.2: 23

Quint. inst. 8.3.62: 309

\section{Seneca}

Sen. benef. 4.19.2: 133

Sen. dial. 1.1 (= De providentia 1.1): 97

Sen. dial. 2-3 (= De providentia 1.2-3): 159

Sen. dial. 3.19.1-2 (= De ira 1.19.1-2): 275

Sen. nat. 2.1.2: 55

Sen. nat. 3.29.1: 8

Sen. nat. 6.14.1-4: 217

Sen. nat. 6.24.6-25.4: 217

Sen. nat. 6.29.1-2: 219

Sen. Thy. 562: 279

Sen. Troad. 449-451: 274

\section{Senofane}

D.-K. 21 A29: VII

D.-K. 21 A33: VII, 7

D.-K. 21 A34: VII

\section{Senofonte}

Xen. mem. 1.4.4-5: 151

Xen. mem. 1.4.6-17: 97

\section{Servio}

Serv. in buc. 4.10 (= Nigid. de diis fr. 67

Swoboda): 24

Serv. in georg. 2.336: 139

Serv. in Aen. 6.72: 22

Serv. in Aen. 6.222: 284

Serv. in Aen. 11.64: 284

\section{Sofocle}

Soph. Aj. 131-132: 113

Soph. Ant. 11-14: 112

Soph. Oed. Col 1224-1227: 161

Soph. OT 4-5: 318-319

\section{Stazio}

Stat. Achill. 1.439: 278 


\section{Strabone}

Strab. geogr. 1.2.8: 231

Strab. geogr. 1.3.16: 231

Strab. geogr. 17.1.36: 132, 325

\section{Stoicorum Veterum Fragmenta (SVF)}

SVF 1.98 (= 19.5 BS): IX, 11

SVF 1.99 (= 20.7 BS): 41, 47, 52, 58

SVF 1.106: IX

SVF 1.107 (= $15.1 \mathrm{BS}): 11$

SVF 1.108: 139

SVF 1.120 (= 15.10 BS): $\quad 45,49$

SVF 1.121: 57

SVF 1.497 (= 15.5 BS): 11

SVF 1.499: IX

SVF 1.500: IX

SVF 1.501: IX, 56

SVF 1.502: IX

SVF 1.503: IX

SVF 1.504 (= $15.12 \mathrm{BS}): \quad$ IX

SVF 1.510 (= $15.11 \mathrm{BS}): \quad$ IX

SVF 1.537 (= 17.2 BS): 117

SVF 2.430: 12

SVF 2.434: 43

SVF 2.458 (=12.7-8 BS): 61

SVF 2.500: 58

SVF 2.528 (= 12.4 BS): $12-13$

SVF 2.549: 47

SVF 2.551: $13,51,58,139$

SVF 2.554: $47,50,58$

SVF 2.555: $58-59$

SVF 2.572: 12

SVF 2.574: $X$

SVF 2.590: 12

SVF 2.596 (= 18.7 BS): 12

SVF 2.603: 12

SVF 2.613: IX

SVF 2.617: 12

SVF 2.620 (= $18.13 \mathrm{BS}): 12$

SVF 2.624 (= 19.18-20 BS): X

SVF 2.625 (= 19.6 BS): XI, 12

SVF 2.632: $X$

SVF 2.636 (= 30.29 BS): 12

SVF 2.652: 46

SVF 2.659: 46

SVF 2.1005: 158

SVF 3.256 (= 26.28 BS): 158
SVF 3.574: XI

SVF 3.585: XI

\section{Svetonio}

Suet. Aug. 31: 22

Suet. Aug. 94: 24

Tacito

Tac. ann. 11.7: 280

Teofrasto

Theophr. fr. 184 FHS\&G: 17-18, 164-190, 246

\section{Teognide}

Thgn. 425-428: 161

\section{Terenzio}

Ter. Andr. 96-97: 277

Ter. Eun. 1029: 279

Ter. Haut. 62-74: 273

\section{Tucidide}

Thuc. 2.47-53: 238

\section{Varrone}

Varro De vita populi Romani fr. 121 Riposati (= 115 Pittà): 23

Varro Men. fr. 84 Ast.: 19, 286

Varro Men. fr. 163 Ast.: 289

Varro Men. fr. 222 Ast.: 285

Varro Men. fr. 225 Ast.: 23

Varro Men. fr. 351 Ast.: 196

Varro Men. fr. 437 Ast.: 19

Varrone Atacino

Varro At. fr. 10 Morel: 189

\section{Virgilio}

Verg. georg. 2.490-492: 255, 305

Verg. Aen. 1.278-279: XXI, 20

Verg. Aen. 1.410: 278

Verg. Aen. 6.127: 328

Verg. Aen. 6.222: 184

Verg. Aen. 7.292: 274

Verg. Aen. 7.421: 276

Verg. Aen. 8.406-416: 281 
Vitruvio

Vitr. 10.1.1-4: 114 